\title{
EVALUACIÓN DEL ESTADO DE LA POBLACIÓN DE CABRA MONTÉS DE LOS MONTES DE TOLEDO: RELACIONES CON EL CIERVO
}

\author{
P. ACEVEDO ${ }^{1,2^{*}}$, J. P. SANTOS ${ }^{2,3}$, R. REAL ${ }^{1} \&$ J. VICENTE ${ }^{2}$ \\ 1 Departamento de Biología Animal, Facultad de Ciencias, Universidad de Málaga, 29071 Málaga \\ *pacevedo@irec.csic.es; pacevedo@uma.es \\ 2 Instituto de Investigación en Recursos Cinegéticos (CSIC-UCLM-JCCM), 13071 Ciudad Real \\ 3 Departamento de Biologia/CESAM, Universidade de Aveiro, 3810-193 Aveiro, Portugal
}

\begin{abstract}
Iberian wild goat subsp. Capra pyrenaica victoriae inhabits fewer and smaller areas than subs. C. p. hispanica reason why the former is considered as Vulnerable by IUCN. Therefore studies aimed to assess the status of C. p. victoriae are very valuables. We studied the habitat use, parasite excretion rates and diet quality of the Montes de Toledo's C. $p$. victoriae population. The red deer is a potential competitor of the Iberian wild goat and subsequently was included in this study. Our results showed that both species had a good population status (good corporal condition, high quality diet and suitable population density). According to the indicators considered in this study we do not expect that red deer suppose a relevant risk for the viability of the Iberian wild goat population. The implications of the results to Iberian wild goat management are deeper discussed.
\end{abstract}

Keywords: Iberian wild goat; diet quality; red deer; bronchopulmonary nematodes; habitat use.

RESUMEN.- La Capra pyrenaica victoriae presenta pocas poblaciones y éstas ocupan áreas reducidas, siendo considerada por ello por la IUCN como vulnerable. Son necesarios, por tanto, estudios locales encaminados a evaluar el estado de dichas poblaciones. En este trabajo estudiamos la población presente en los Montes de Toledo a través del análisis del uso que hace del hábitat, del nivel de parasitación y de la calidad de su dieta. El ciervo fue también incluido en este esquema de monitorización por ser un potencial competidor para la cabra montés. Los resultados indican que ambas especies presentan un adecuado estado poblacional (buena condición corporal, dieta de elevada calidad y valores de densidad razonables). Según los indicadores considerados no sería esperable que el ciervo supusiera un riesgo relevante 
Palabras clave: Cabra; calidad de dieta; ciervo; parásitos; uso del hábitat.

\section{Introducción}

Las poblaciones de caprinos silvestres son particularmente vulnerables a la extinción debido a su aislamiento genético, sus requerimientos de hábitat y su reducida tasa reproductiva (Shackleton, 1997). Además de éstos, la cabra montés (Capra pyrenaica) se ve afectada por otros factores de riesgo adicionales como son el incremento del ganado doméstico y ungulados exóticos, que podrían transmitir enfermedades a la fauna nativa (ver Gortázar et al., 2006) y competir por recursos (Acevedo et al., 2007a, 2007b; Acevedo \& Cassinello, 2009a), y la presión turística (R.C. Soriguer, comunicación personal), que tendrán efectos más marcados cuanto peor sea el estatus de las poblaciones. Lamentablemente este hecho ha quedado recientemente corroborado con la extinción, a finales de los 90, de la subespecie C. p. pyrenaica que habitaba el Pirineo (García-González \& Herrero, 1999).

La cabra montés estaba representada en la Península Ibérica por 4 subespecies de las que sólo 2 permanecen en la actualidad, la C. $p$. victoriae, vinculada al noroeste, y la C. $p$. hispanica que se distribuye por el arco montañoso del este peninsular (Figura 1). La reducida área de distribución es una de las razones por las que la IUCN considera Vulnerable (VUd2) a la subespecie del noroeste, que "presenta pocas poblaciones y éstas ocupan áreas reducidas" (IUCN, 2007). Sin embargo, la situación de la otra subespecie es bien distinta, con distribución amplia y un elevado número de núcleos poblacionales, razones por las que la IUCN la considera como de Bajo Riesgo, aunque próxima a considerarse Vulnerable (LR/nt; IUCN, 2007). Recientemente la IUCN ha cambiado el estatus de la especie considerándola en el grupo de Preocupación Menor al detectar un incremento de sus poblaciones (IUCN, 2009). Pese a ello, la situación de ambas subespecies es claramente distinta, siendo la C. p. victoriae la que muestra un estado más crítico debido a la vulnerabilidad de sus poblaciones (Escós et al., 2008; Acevedo \& Cassinello, 2009b; Acevedo \& Real, en prensa). Así, la subespecie C. p. victoriae se distribuye en sólo 7 de los ya más de 50 núcleos poblacionales presentes en la Península Ibérica (Pérez et al., 2002).

Por otro lado, estudios recientes han evidenciado que, a escala peninsular, las poblaciones de la subespecie $C$. $p$. victoriae se encuentran restringidas a 


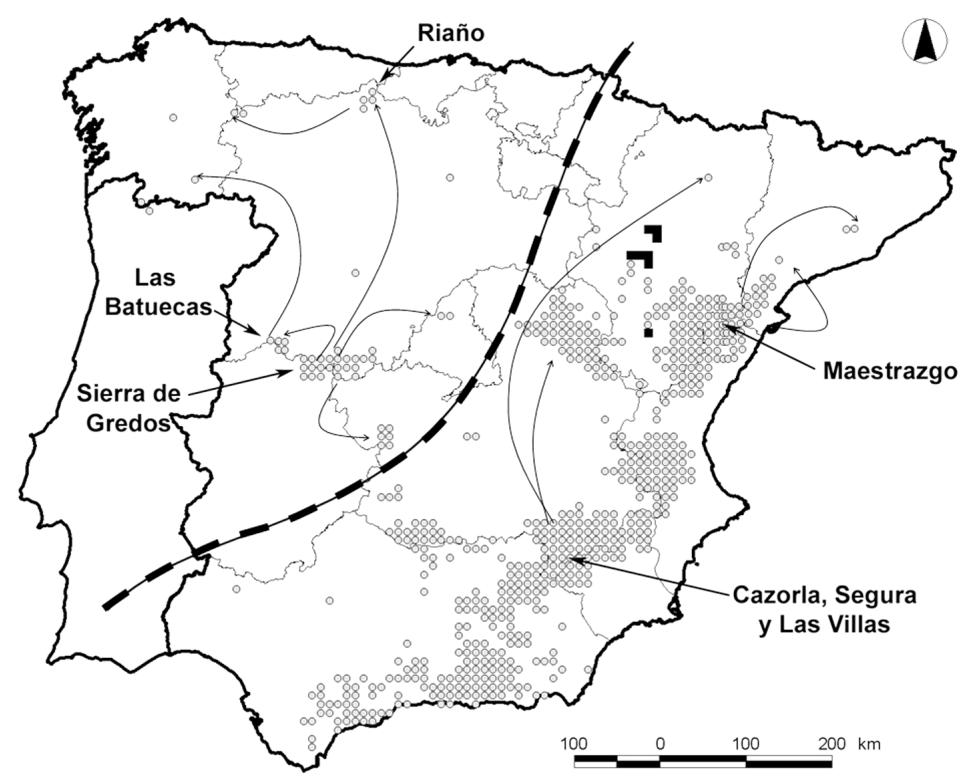

Figura 1. Distribución actual de la cabra montés en la Península Ibérica. La línea discontinua divide la distribución de ambas subespecies, al noroeste la C. $p$. victoriae, y en el este y el sur la C. $p$. hispanica. Las flechas muestran translocaciones. Adaptada de Acevedo \& Cassinello (2009b)

Figure 1. Current distribution of Iberian wild goat in the Iberian Peninsula. Grey lines delimit boundaries of the provinces. The distribution of the two subspecies is represented by the discontinuous line, C. p. victoriae in the north-west, and C. p. hispanica in the south and east. Arrows indicate translocations from labelled populations. Figure adapted from Acevedo \& Cassinello (2009b).

determinados enclaves dentro de áreas ambientalmente adecuadas de mayor amplitud (Acevedo \& Real, en prensa). Esto podría estar mostrando la existencia de algunos factores, bióticos u abióticos, que podrían estar limitando la accesibilidad de las monteses a otras áreas favorables que permanecen deshabitadas. Así, estudios realizados a escala local, y encaminados a determinar el estado de las poblaciones, aportarán información de utilidad para comprender los mecanismos que regulan la dinámica de esta subespecie.

Aunque no se han realizado estudios genéticos que lo corroboren, la escasa información disponible indica que la población de cabra montés del Parque Nacional de Cabañeros pertenece a la subespecie C. p. victoriae. Así, se tiene constancia de introducciones realizadas antes de 1995 (Pérez et al., 2002) con animales procedentes supuestamente de Gredos. En este mismo sentido, 
análisis biogeográficos realizados recientemente son congruentes con dicha procedencia ya que muestran que las cabras monteses de los Montes de Toledo ocupan áreas ambientalmente más próximas a las que ocupan las $C$. $p$. victoriae de Gredos o Riaño que a las zonas ocupadas por C. $p$. hispanica en Sierra Morena o en el Alto Tajo (Acevedo \& Real, en prensa).

Pérez et al. (2002), basándose en información proveniente de conversaciones personales con trabajadores del Parque, describen que las cabras en este territorio se distribuyen por las sierras de Las Parrillas, Muelas y Ciguiñuelas. Estos mismos autores estimaron una población de 15-20 ejemplares para este núcleo en 1999. Posteriormente, un muestreo realizado en 2003 ha confirmado la presencia de la especie en las inmediaciones del Pico Rocigalgo (ver Acevedo et al., 2005). Dicho muestreo mostró una reducida abundancia relativa en este núcleo en comparación con otras poblaciones de cabra montés de Castilla-La Mancha (CLM).

Además de estimar la abundancia relativa de las poblaciones en el muestreo realizado en 2003 se realizó un estudio parasitario de las poblaciones de cabra de CLM. Las prevalencias obtenidas para la población de Montes de Toledo fueron más elevadas de lo que cabía esperar dada la abundancia relativa de cabra montés en este núcleo. Así el $72 \%$ de las muestras analizadas presentaron formas de excreción de la Familia Protostrongylidae y el 33\% de Dictyocaulus spp. (Acevedo et al., 2005). Esto, considerando la clara densodependencia que muestran estos grupos de parásitos en este hospedador, podría indicar que las abundancias estimadas entonces pudieran estar infravalorando la abundancia real de la población.

En este contexto, el objetivo principal de este estudio es conocer el estado de la población de cabra montés presente en el Parque Nacional de Cabañeros, y estudiar el uso que ésta hace del hábitat. Esto permitirá la implantación de un sistema de seguimiento de dicha población a lo largo del tiempo. Además, se cree necesario incorporar al ciervo en este sistema de seguimiento para así poder detectar las posibles relaciones interespecíficas que medien la simpatría de ambas especies.

\section{Metodología}

\subsection{Encuestas de distribución}

Son numerosos los trabajos en los que se han empleado datos provenientes de encuestas para conocer la distribución y tendencias poblacionales de ungulados cinegéticos (Gortázar et al., 2000). Así, en este estudio se han distribuido encuestas con el fin de recopilar información sobre la población de 
cabra montés presente en el Parque Nacional de Cabañeros y sus inmediaciones (área de estudio). En las encuestas se ha solicitado información cualitativa sobre la procedencia y características del núcleo fundador de la población, y también sobre la percepción personal de la tendencia que ésta ha experimentado en los últimos años. Además de los formularios, las encuestas han ido acompañadas de mapas de la zona donde se les pedía a los colaboradores que señalasen los parajes en los que suelen avistar cabra montés.

\subsection{Uso del hábitat y densidad poblacional}

Los muestreos de excrementos en parcelas pueden ser usados para estudiar los patrones de uso y preferencias de hábitat en ungulados (Edge \& Marcum, 1989; Hemami et al., 2005). Actualmente están disponibles nuevas herramientas, basadas en sistemas de posicionamiento global (GPS), que permiten realizar estudios más precisos (Moen et al., 1997), pero lamentablemente éstas no están todavía al alcance de todos los proyectos de investigación. Por otro lado, el recuento de excrementos en parcelas es un método usado para estimar la abundancia de las poblaciones de ungulados (Acevedo et al., 2008, 2010), siendo posible incluso estimar valores de densidad siempre que la tasa de deposición de excrementos y el tiempo de permanencia de los excrementos en el medio sean parámetros conocidos (Smart et al., 2004). En este trabajo, mediante el recuento de excrementos en parcelas se han estudiado los patrones de uso del hábitat de cabras monteses y ciervos dentro del área de distribución de la cabra montés en el Parque a lo largo de un ciclo anual (desde julio de 2009 hasta junio de 2010).

Una vez determinada el área de distribución de la cabra montés en el Parque, a lo largo de 4 recorridos se fijaron 32 parcelas sobre las que realizar el recuento de excrementos (Figura 2), aplicando un esfuerzo de muestreo aproximado de 4 parcelas por cada 500 ha de territorio. De estos 4 recorridos, 2 de ellos discurrían por zonas con cierto manejo cinegético que se manifestaba en el aporte de alimentación suplementaria. De acuerdo a experiencias previas con esta metodología en poblaciones de ciervo (Acevedo et al., 2008) y corzo (Acevedo et al., 2010), y dada la reducida densidad esperada de cabra montés en este núcleo poblacional, las parcelas han sido de $5 \mathrm{~m} \times 5 \mathrm{~m}$, es decir de $25 \mathrm{~m}^{2}$ (se han muestreado $800 \mathrm{~m}^{2}$ ).

Todas las parcelas han sido revisadas con una periodicidad que ha variado entre los 42 y los 55 días, con la excepción de los meses de invierno en los que las condiciones climáticas adversas no permitieron hacer una de las revisiones. En cada revisión se ha registrado el número de grupos fecales de cada una de las especies consideradas -cabra montés y ciervo- presentes en cada 


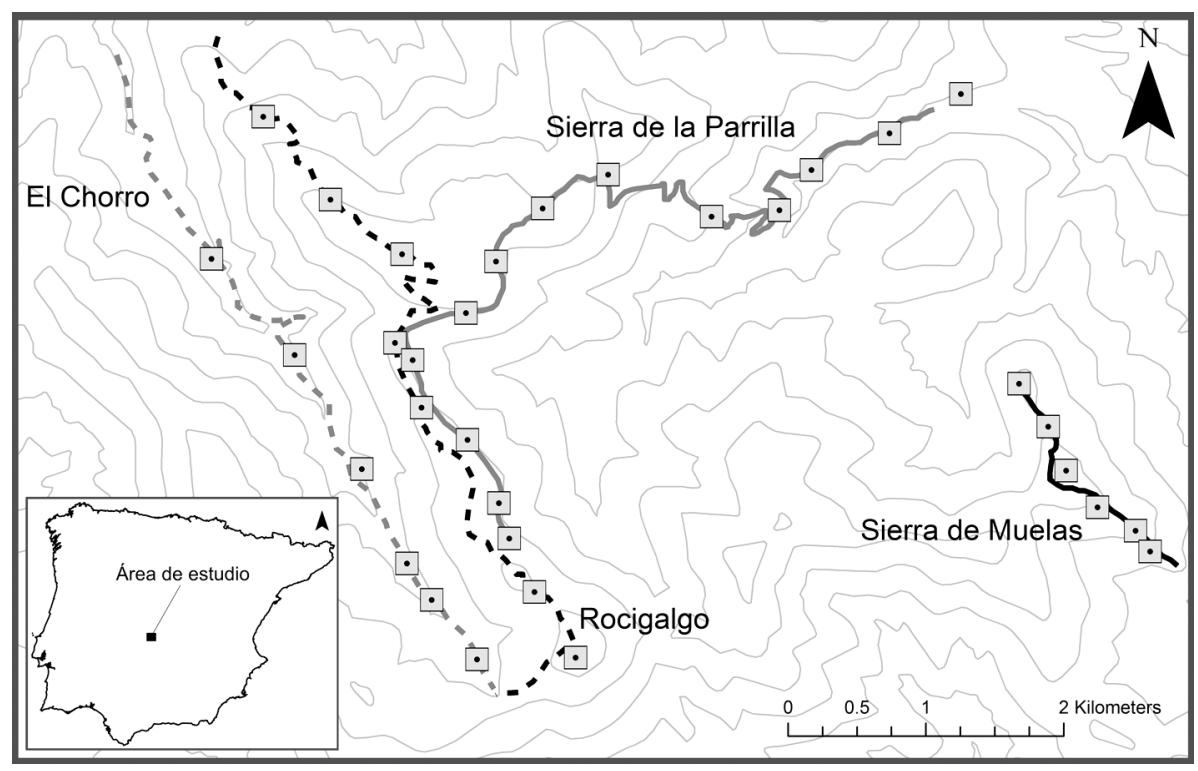

Figura 2. Localización del área de estudio y de las parcelas de recuento de excrementos a lo largo de los recorridos diseñados, dos de ellos por zonas sin manejo cinegético (líneas discontinuas) y dos por zonas manejadas (líneas continuas). Las curvas de nivel (cada $200 \mathrm{~m}$ ) son incluidas para mostrar la orografía del área de estudio.

Figure 2. The location of sampling plots in which Iberian wild goat population was monitored from faeces. Unmanaged (discontinuous lines) and intensively managed (continuous lines) areas are differentiated. The orography of the study area is shown with the elevation model.

parcela. Tras el recuento, los excrementos eran eliminados de las parcelas, por lo que se ha usado el Faecal Accumulation Rate (Smart et al., 2004) como índice de abundancia. A partir de este método se puede inferir un valor de densidad poblacional siempre que se conozca la tasa de defecación de estas especies. Para el caso del ciervo, en este tipo de ambientes mediterráneos, puede considerarse de 11.8 grupos/día (Acevedo et al., 2008), y para la cabra, aunque desconocida, podría situarse entre la del ciervo y la del corzo, y por tanto tomar valores próximos a 15.0 grupos/día (son 20 para el caso del corzo; Acevedo et al., 2010). Los recuentos de excrementos se han aprovechado para coger muestras de heces frescas con las que realizar estudios parasitarios y de calidad de dieta (ver abajo). 


\subsection{Parásitos y calidad de la dieta}

Con las muestras de excrementos tomadas en el campo y los análisis realizados en el laboratorio $(n=115)$ se ha podido caracterizar la variación anual de excreción de las principales formas parásitas presentes en ambas especies (Vicente et al., 2005). La extracción de larvas L1 de nematodos de las heces fue realizada en menos de 24-48 horas tras su recolección. A partir de 8-10 gramos de heces, se empleó el método Baermann de extracción larvaria modificado por Forrester \& Lankester (1997). Este método, consiste en inducir la migración de las larvas en agua en un vaso de precipitados de $250 \mathrm{ml}$ durante 24 horas. Las heces pesadas se colocan envueltas por una tela de vinilo de $2 \mathrm{~mm}$ de retícula cuadrada a modo de sobre grapado. Las larvas fueron cuantificadas en cámara de Favatti y se expresan como larvas por gramo de heces. Las larvas fueron identificadas hasta el nivel de género según su morfología (Melhorn et al., 1992; Cordero del Campillo \& Rojo, 1999; Anderson, 2000).

Por otra parte, la monitorización de la calidad de la dieta puede ofrecer un diagnóstico adicional del estado de la población. Los altos requerimientos de proteína de los animales en relación a su disponibilidad en las plantas, convierten al nitrógeno ingerido en un buen indicador de la calidad proteica de la dieta (Mattson, 1980; Putman, 1984), y por tanto al nitrógeno fecal en un parámetro de gran utilidad como herramienta para la gestión de ungulados silvestres (Massey et al., 1994; Hamel et al., 2009). En este estudio se ha usado el nitrógeno fecal como indicador de la calidad de la dieta. Los análisis para cuantificar el nitrógeno se han realizado sobre los grupos fecales recogidos durante los muestreos de campo siempre que la cantidad de muestra era suficiente para la realización de ambos análisis. Se ha cuantificado el nitrógeno de 163 grupos fecales, entre ciervo y cabra montés. Para ello se ha usado el analizador elemental LECO CHNS-932 que determina de forma cuantitativa el carbono, hidrógeno, nitrógeno y azufre de las muestras. La técnica está basada en la completa e instantánea oxidación térmica de 1 a $3 \mathrm{mg}$ de la muestra mediante una combustión en una atmósfera controlada en presión y enriquecida con oxígeno puro a una temperatura de $1100{ }^{\circ} \mathrm{C}$, generando productos gaseosos, $\mathrm{CO}_{2}, \mathrm{H}_{2} \mathrm{O}, \mathrm{SO}_{2}$ y $\mathrm{NO}_{2}$ que, una vez separados, se cuantifican mediante métodos selectivos de absorción de infrarrojos y de conductividad térmica. Una vez registrados los datos, se procesan junto al peso de la muestra y a los datos proporcionados por una muestra patrón, tras lo cual obtendremos el contenido porcentual de cada elemento de la muestra -en este caso nitrógeno- con respecto al peso analizado. 


\section{Resultados}

\subsection{Encuestas de distribución}

Se han cumplimetado 10 encuestas de distribución realizadas a personal de diversos sectores profesionales, todos ellos con una estrecha vinculación con el medio natural y con el área de estudio. En términos generales, se puede decir que entre los encuestados existe conocimiento de la existencia de la población de cabra montés, pero ésta no es considerada como especialmente relevante por ninguno de los sectores encuestados, entre los que se incluyen lugareños, personal técnico y guardas de caza de fincas próximas.

Todos los encuestados que aportaron información sobre el origen de la población señalaron que la procedencia de los animales fue Gredos. El $66 \%$ de los encuestados considera que la población de cabra montés objeto de estudio presenta una tendencia al aumento de sus efectivos, siendo esta percepción más evidente en los últimos años. El porcentaje restante perciben estabilidad y ninguno de ellos disminución. El tamaño máximo de grupo visto por los colaboradores fue de 8 ejemplares y el 100\% de los encuestados avistaron hembras con crías.

\subsection{Uso del hábitat y densidad poblacional}

Con las 7 revisiones realizadas de las 32 parcelas de recuento de excrementos se han podido detectar 775 grupos fecales de ciervo y 108 grupos fecales de cabra montés. El número medio de grupos detectados por revisión -excluyendo los datos de la primera en la que se limpiaron las parcelas- fue de $78 \pm 19$ grupos fecales de ciervo y $8 \pm 2$ de cabra montés, no apreciándose diferencias estadísticamente significativas entre revisiones, ni en el caso del ciervo (Kruskal-Wallis: $\left.\mathrm{Chi}^{2}=4.39,5 \mathrm{gl}, \mathrm{p}=0.495\right)$, ni en el de la cabra montés (Kruskal-Wallis: $\mathrm{Chi}^{2}=4.91,5 \mathrm{gl}, \mathrm{p}=0.427$ ).

En relación a los diferentes recorridos considerados en el estudio, se han encontrado diferencias significativas en lo que respecta al índice de abundancia, medido como el número medio de grupos fecales detectados por parcela y mes, para el caso del ciervo (Kruskal-Wallis: $\mathrm{Chi}^{2}=26.72,3 \mathrm{gl}, \mathrm{p}<0.001$ ), sin embargo las diferencias no fueron significativas para el caso de la cabra montés, mostrándose que esta especie presenta una abundancia similar en todos ellos (Kruskal-Wallis: $\mathrm{Chi}^{2}=3.81,3 \mathrm{gl}, \mathrm{p}=0.283$ ).

El uso que cada una de las especies hace de las parcelas ha sido modelado (modelos lineales generalizados con distribución de Poisson y función de vín- 
culo logarítmica) con variables relacionadas con la orografía y los usos del suelo (Tabla 1). El modelo indica que la pendiente del terreno es determinante del uso que los ciervos hacen del territorio, registrándose un mayor uso de

Tabla 1. Resultados de los modelos lineales generalizados realizados para analizar el efecto de variables ambientales sobre el uso, a nivel de parcela, que cada especie hace del territorio. Table 1. Variables retained in the generalized lineal models developed to explain the red deer and Iberian ibex habitat use at plot level.

\begin{tabular}{|l|c|c|c|}
\hline Variable & Wald & $g l$ & $p$ \\
\hline (Intersección) & 240.328 & 1 & $<0.001$ \\
Recorrido & 57.373 & 3 & $<0.001$ \\
Orientación & 56.427 & 6 & $<0.001$ \\
Vegetación & 45.287 & 4 & $<0.001$ \\
Pendiente & 13.485 & 1 & 0.001 \\
\hline \multicolumn{4}{|c|}{ Ciervo } \\
\hline (Intersección) & 0.430 & 1 & 0.512 \\
Recorrido & 1.302 & 2 & 0.522 \\
Orientación & 4.899 & 5 & 0.428 \\
Vegetación & 17.424 & 4 & 0.002 \\
Pendiente & 0.604 & 1 & 0.437 \\
\hline \multicolumn{4}{|l}{}
\end{tabular}

las parcelas con menor pendiente. Además, los resultados indican que los ciervos son más frecuentes en aquellas parcelas situadas en orientación suroeste y menos en las zonas de bosque claro (ver Figura 3). Además se han detectado diferencias significativas entre recorridos. Por su parte, el modelo realizado para la cabra montés muestra que no existen diferencias entre fincas, ni entre tipos de orientación, ni en relación a la pendiente. Sólo se observaron diferencias significativas en el uso en relación al tipo de vegetación, siendo las zonas de vegetación rala y matorral arbolado las más frecuentadas por las monteses, y los bosques claros los menos usados (ver Figura 3).

En base al recuento de excremento se ha podido estimar una densidad media de ciervo para todo el área de estudio de $14.62 \pm 2.27 \mathrm{ind} / \mathrm{km}^{2}$, y de $1.39 \pm 0.17 \mathrm{ind} / \mathrm{km}^{2}$ para la cabra montés. Por tanto, considerando que el área de estudio son aproximadamente 4250 ha, el tamaño de la población de cabra montés objeto de estudio se podría situar entre los 52 y 66 individuos. 

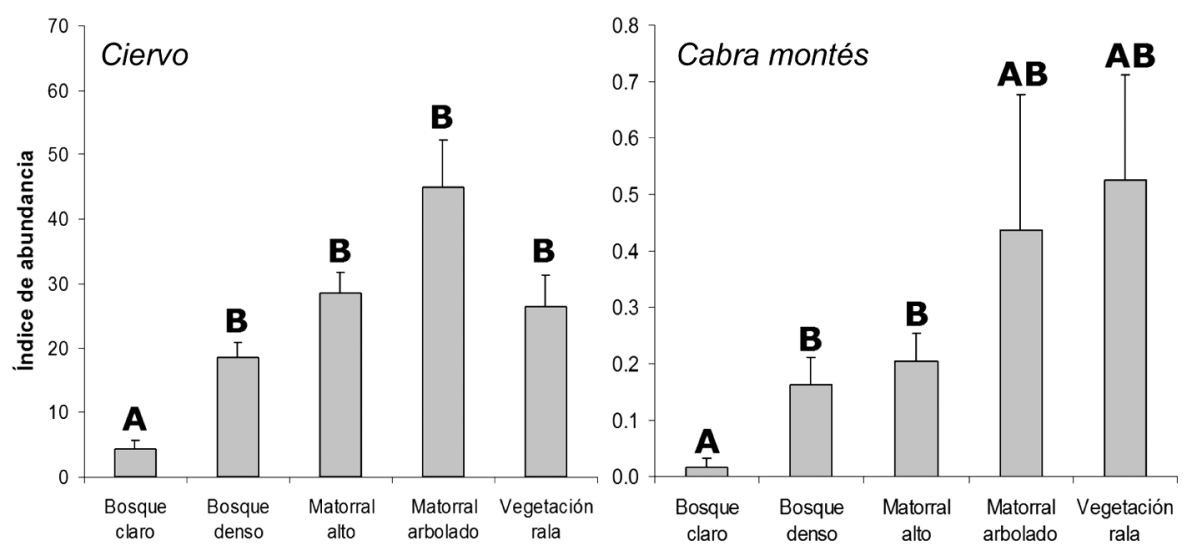

Figura 3. Valores medios de abundancia de ciervo y cabra montés en las parcelas en función de la vegetación. Los valores son las medias marginales de la variable dentro del modelo (Tabla 1). Las barras de error representan el error típico. Letras distintas dentro de cada gráfico indican diferencias significativas.

Figure 3. Mean values (SE) of abundance for each species -red deer and Iberian wild goat-in the plots in relation to vegetation. Same capital letter: did not differ significantly $(p>0.05)$.

\subsection{Parásitos y calidad de la dieta}

El $96.43 \%$ de los grupos fecales de ciervo analizados $(\mathrm{n}=84)$ presentaron larvas de Elaphostrongylus cervi y en un $13.09 \%$ de los mismos se identificaron formas de excreción de Dictyocaulus spp. Con las muestras de cabra montés $(\mathrm{n}=31)$ se obtuvo una prevalencia también elevada, $70.97 \%$ para las formas de excreción de la familia Protostrongylidae, y una $12.90 \%$ para Dictyocaulus spp. Los resultados de los modelos lineales generalizados (distribución binomial negativa y función de vínculo logarítmica), realizados para explorar los factores relacionados con las tasas de excreción de los parásitos de la familia Protostrongylidae (Tabla 2), evidencian que para ambas especies existen diferencias significativas entre recorridos y estaciones (Figura 4), presentando ciervo y cabra un patrón estacional inverso.

El porcentaje medio de nitrógeno en grupos fecales de ciervo $(n=113)$ fue $2.18 \pm 0.41 \%$ y de cabra montés $(n=50)$ fue $2.23 \pm 0.41 \%$. Los resultados de los modelos lineales generales (distribución de normal y función de vínculo identidad) se muestran en la Tabla 3. El modelo indica que los niveles de nitrógeno en cabra montés son significativamente superiores a los detectados en ciervo. Independientemente de la especie, se han encontrado diferencias 
Tabla 2. Resultados de los modelos lineales generalizados realizados para analizar el efecto de finca y estación sobre la abundancia de protostongílidos.

Table 2. Variables retained in the generalized lineal models developed to explain the excretion rates of Elaphostrongylus cervi and Protostrongylidae.

\begin{tabular}{|l|c|c|c|}
\hline Variable & Wald & $g l$ & $p$ \\
\hline (Intersección) & 766.286 & 1 & $<0.001$ \\
Recorrido & 7.964 & 3 & 0.047 \\
Estación & 32.359 & 2 & $<0.001$ \\
\hline \multicolumn{4}{|c|}{ Ciervo - E. cervi } \\
\hline (Intersección) & 4.687 & 1 & 0.030 \\
Recorrido & 11.000 & 3 & 0.012 \\
Estación & 8.258 & 2 & 0.016 \\
\hline \multicolumn{4}{|c|}{ Cabra-Protostrongylidae } \\
\hline
\end{tabular}

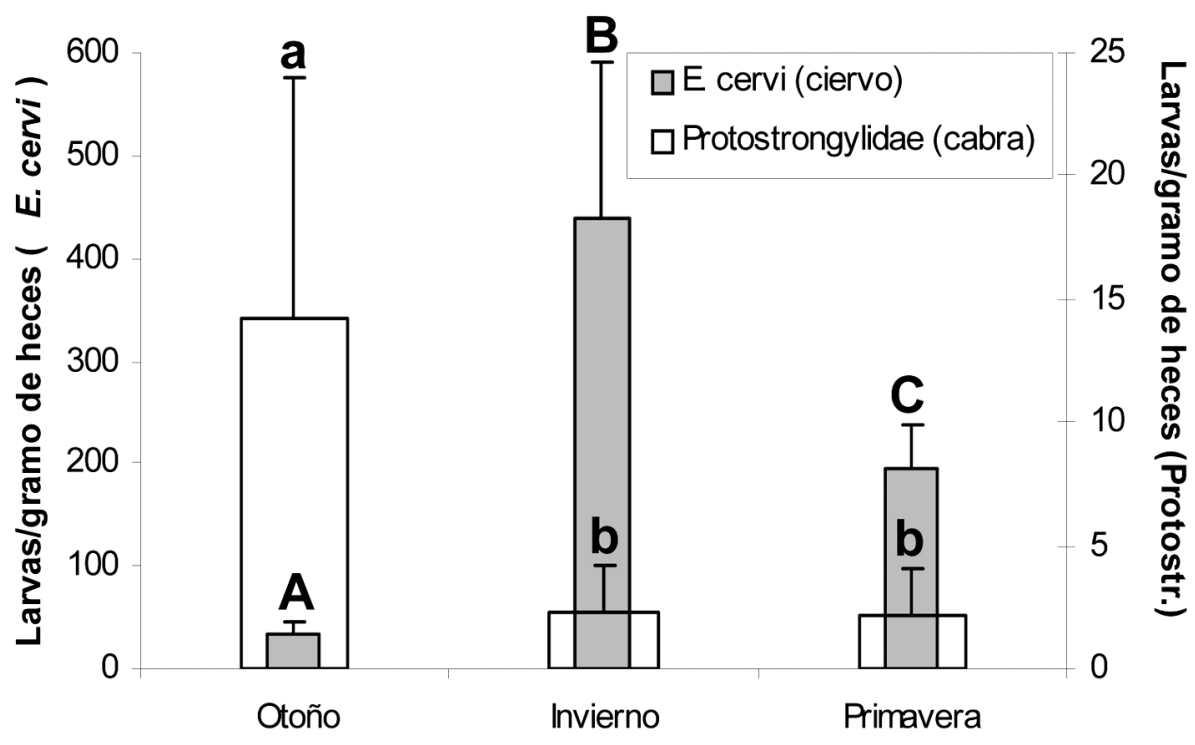

Figura 4. Diferencias entre estaciones y especies en las tasas de excreción de parásitos protostrongílidos. Los valores son las medias marginales de las variables dentro del modelo (Tabla 2). Las barras de error representan el error típico. Letras distintas, mayúsculas (ciervo) y minúsculas (cabra), indican diferencias significativas.

Figure 4. Seasonal patterns of Protostrongylidae's excretion rates in both red deer and Iberian wild goat. Same capital letter or lower case letter: did not differ significantly ( $p>0.05)$. 
significativas entre estaciones y entre recorridos con y sin manejo cinegético. Así, los mayores niveles se detectaron en primavera y en aquellos recorridos en los que el manejo cinegético es inexistente (Figura 5).

Tabla 3. Resultados de los modelos lineales generalizados realizados para analizar el efecto de especie, fincas (manejadas o no manejadas) y estación sobre los niveles de nitrógeno fecal.

Table 3. Variables retained in the generalized lineal models developed to explain the individual levels of faecal nitrogen.

\begin{tabular}{|l|c|c|c|}
\hline Variable & $F$ & $g l$ & $p$ \\
\hline (Intersección) & 4879.621 & 1 & $<0.001$ \\
Especie & 2.815 & 1 & 0.045 \\
Estación & 34.022 & 3 & $<0.001$ \\
Recorrido & 4.256 & 1 & 0.041 \\
Especie*Estación & 1.399 & 3 & 0.245 \\
Especie*Recorrido & 3.878 & 1 & 0.045 \\
\hline
\end{tabular}

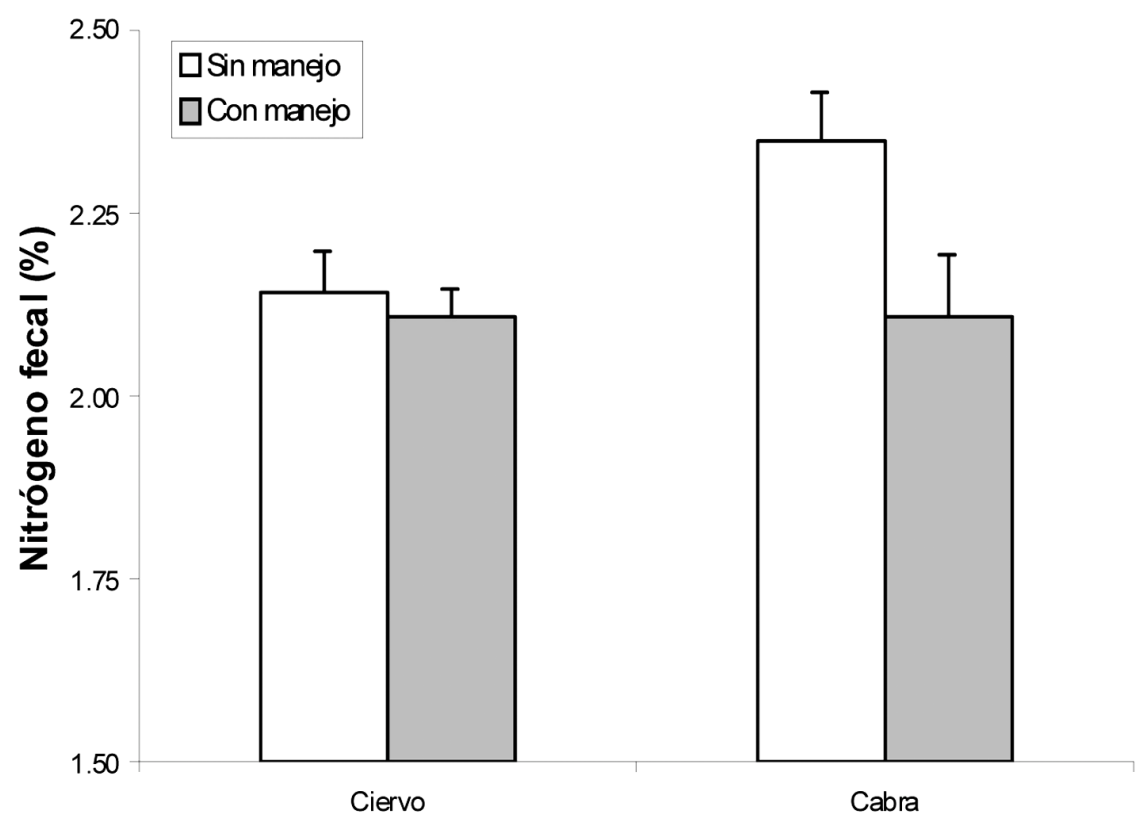

Figura 5. Diferencias entre especies y recorridos (en relación al manejo cinegético) en los niveles de nitrógeno fecal. Los valores son las medias marginales de la interacción entre estas variables dentro del modelo (Tabla 3). Las barras de error representan el error típico.

Figure 5. Mean values (SE) of faecal nitrogen levels in relation to game management and monitored species -red deer and Iberian wild goat-. 


\section{Discusión}

\subsection{Sobre la población en general}

Los estudios realizados han permitido realizar una primera caracterización, en función de ciertos indicadores biológicos, del estado de la población de cabra montés presente en el Parque Nacional de Cabañeros. Mediante las encuestas de distribución se ha podido corroborar que dicha población parece tener su origen en introducciones realizadas con animales procedentes de Gredos (Pérez et al., 2002). En general, los encuestados perciben que esta población en la actualidad se encuentra en aumento, si bien es cierto que no es un crecimiento especialmente acusado a tenor de las densidades observadas. Esta tendencia poblacional es congruente con la descrita para otras poblaciones de cabra montés de CLM (Acevedo et al., 2007a) y otras poblaciones peninsulares de esta subespecie (Escós et al., 2008).

En el caso de la población de Cabañeros, su crecimiento podría estar ralentizado por la presencia de elevadas densidades de ciervo, pero son muchas las causas que pueden estar restringiendo la expansión numérica de este núcleo poblacional y permanecen desconocidas. Escós et al. (2008) analizando la evolución temporal de varias poblaciones de cabra montés, mostraron que todas las poblaciones de C. p. hispanica están en notoria expansión numérica, pero la población de C. $p$. victoriae monitorizada, la de Gredos, se mantiene estable en los últimos años. Esto indica que podrían existir razones ecológicas diferentes a la competencia que explicaran que las poblaciones de esta subespecie no tuvieran la misma potencialidad que la C. $p$. hispanica para expandirse. Los estudios parasitarios y de calidad de dieta realizados aquí, aunque no son conclusivos, aportan un poco de luz sobre los efectos de esta relación interespecífica (ver abajo).

\subsection{Uso del hábitat y densidad poblacional}

Los modelos realizados para determinar el uso que los ciervos y las cabras hacen del territorio han mostrado diferencias claras entre ambas especies. Así, el ciervo parece que hace un uso acorde con sus requerimientos naturales seleccionando zonas calientes (orientación SW), de matorral arbolado y con poca pendiente (ver Acevedo \& Cassinello, 2009a), mientras que el uso que hace la cabra montés indica que esta especie es más frecuente en zonas de vegetación escasa (Acevedo et al., 2007a). Aunque con este tipo de estudios es difícil inferir competencia entre especies, la segregación observada podría 
estar indicando que i) ambas especies presentan diferentes requerimientos ambientales - poco probable dadas las similitudes ecológicas previamente comentadas-, pero también que ii) las cabras se han quedado relegadas a aquellos ambientes poco frecuentados por los ciervos. Los datos disponibles no permiten inferir cual de estas causas puede ser la que esté mediando el uso que las monteses hacen del territorio, siendo necesarios para ello estudios basados en el radioseguimiento de los animales de ambas especies junto con estudios de dieta realizados para ambas especies en este territorio.

La densidad media de ciervo en el área de estudio puede considerarse media, o incluso media-alta, en relación a otras fincas de caza de CLM (Acevedo et al., 2008). Como anteriormente se ha comentado, elevadas densidades de ciervo podrían determinar la tendencia de las poblaciones de cabra montés. Esta misma interpretación podría también extraerse del artículo de Pérez et al. (2002) en los que muestran que las poblaciones de cabra montés en general están en aumento, con la salvedad de la población de Sierra Morena que es precisamente la que convive con densidades elevadas -en ocasiones por encima de la capacidad de carga del medio- de ciervo. La densidad de cabra montés en el área de estudio se puede considerar baja en relación a las estimadas para otras poblaciones manchegas de las cuencas de los ríos Segura y Mundo en la provincia de Albacete. Trabajos realizados en 2006 en dichas poblaciones han mostrado densidades que oscilaban entre 6 y 15 ind $/ \mathrm{km}^{2}$ (Acevedo et al., 2007c), siendo estos valores notoriamente más elevados que los de la población de Cabañeros.

\subsection{Parásitos y calidad de la dieta}

Los resultados indican que la prevalencia media de E. cervi detectada en las poblaciones de estudio es elevada (media 96.43\%) y congruente con la obtenida en estudios previos realizados sobre estas mismas poblaciones (ver Vicente et al., 2006), y también sobre otras poblaciones de similares características en cuanto a manejo y área geográfica (Tayce et al., 2008). En el marco de estos estudios se puede decir que las poblaciones estudiadas se sitúan entre el $25 \%$ de las poblaciones con prevalencias más elevadas de una selección de 18 de este mismo entorno (Vicente et al., 2006). La clara vinculación existente entre la prevalencia de este nemátodo y la condición corporal de los ciervos (Vicente et al., 2007) indica que estas poblaciones presentan una buena condición corporal individual, lo que podría estar relacionado con dos factores principales: i) las poblaciones presentan una densidad adecuada a la potencialidad del medio, y ii) en el caso de presentar una densidad más elevada, las poblaciones son manejadas con el fin de mitigar los efectos negati- 
vos que la sobreabundancia poblacional tiene sobre la condición corporal de los individuos.

En lo que respecta al ciclo de excreción anual (Figura 4), los resultados de este estudio destacan la presencia de un pico de excreción en el invierno (Vicente et al., 2005). Los picos estacionales pueden ser interpretados como una respuesta del parásito, más o menos rápida, a las condiciones meteorológicas manteniéndose el máximo anual durante el periodo de lluvias. Los resultados aquí mostrados son coherentes con investigaciones anteriores y ponen de manifiesto que el periodo crítico para estos animales en estas latitudes no se centra en el invierno tal y como ocurre en otras poblaciones del norte de Europa, por ejemplo Polonia (Demiaszkiewicz, 1987), aquí es el verano (principios de otoño) el periodo de menor disponibilidad de alimento.

Por otro lado, la prevalencia de Protostrongylidae obtenida en las muestras de cabra montés fue también elevada $(71 \%)$ y muy similar a las obtenidas en el muestreo realizado en 2003 (70\%; Acevedo et al., 2005). Al igual que anteriormente se ha comentado para el caso de los ciervos, las tasas de excreción detectadas en las muestras de cabra son elevadas si se contextualizan en el marco de otras poblaciones de CLM. Podemos decir que la población de estudio está entre el $30 \%$ de las poblaciones con prevalencias más elevadas de una selección de 14 fincas de este mismo entorno (Acevedo et al., 2005). Otros autores han estudiado las tasas de infestación y excreción de protostongílidos en poblaciones de cabra montés de Andalucía y han obtenido prevalencias incluso más elevadas que las presentadas aquí (Alasaad et al., 2009).

En la Figura 4 se muestra la estacionalidad de la excreción de larvas en cabra montés. En ella se muestra una situación claramente distinta, casi opuesta, a la comentada anteriormente para el ciervo. En este caso, el pico de excreción se detecta en otoño y no en invierno. No existen estudios previos sobre la estacionalidad de estos nematodos en cabra montés con lo que no se pueden establecer comparaciones y resulta, por tanto, más complicado realizar una interpretación certera del patrón observado. El patrón podría deberse a que es a finales de otoño cuando ocurre el celo en esta especie por lo que este es el periodo de máxima agregación de la poblaciones, máxime en el área de estudio donde dado el reducido tamaño poblacional es posible que todas las cabras del núcleo se agrupen en 2-3 rebaños en esta época. Sería necesario realizar un estudio continuado en el tiempo -idealmente usando collares GPS- para corroborar estos resultados ya que, al carecer de réplica, por el momento deben ser tomados con cierta cautela.

La cantidad de nitrógeno fecal nos informa sobre la calidad proteica de la dieta de los individuos. Las poblaciones de ciervo estudiadas presentan valores elevados, con un $2.19 \%$ de nitrógeno de media, se sitúan entre el $25 \%$ de las fincas con mayores niveles de nitrógeno (Toledano, 2008). Las de cabra, 
con un $2.32 \%$ de media, presentan niveles ligeramente superiores a todas las poblaciones de ciervo de dicho estudio en el que el máximo valor obtenido fue $2.30 \%$. Por tanto, los resultados parecen estar indicando que las poblaciones estudiadas muestran indicios de disponer de una dieta de buena calidad proteica. Este resultado es congruente con la discusión previamente planteada sobre las tasas de excreción de Protostrongylidae como indicadores de una buena condición corporal de los animales. Al igual que antes, los altos niveles detectados aquí indicarían que la densidad poblacional se ajusta a la capacidad de carga del medio y que, en el caso de sobrepasarla, la alimentación suplementaria estaría mitigando el efecto también a este nivel de estudio.

Tal y como previamente ya ha sido sugerido, la cantidad de nitrógeno excretado en heces de cabra fue significativamente más elevada que el excretado en heces de ciervo, siendo la diferencia entre especies más acentuada en los recorridos sin manejo cinegético (ver Figura 5). Aunque las diferencias entre especies pudieran ser esperables dada la determinación de estrategias alimentarias diferentes en función de su tamaño corporal (Gordon, 1989), ambas especies presentan un elevado solapamiento de dieta (Martínez et al., 1992) por lo que podrían ser igualmente esperables niveles de nitrógeno similares entre ambas especies. En este caso concreto parece más razonable pensar que las diferencias observadas entre especies pudieran estar más relacionadas con el uso del hábitat que con diferencias fisiológicas. Así, los mayores niveles de nitrógeno se han detectado en los recorridos que discurren por terrenos sin manejo cinegético, siendo esto especialmente notorio en el caso de la cabra montés. Este resultado contrasta con estudios previos en los que se pusieron de manifiesto mayores niveles de nitrógeno en fincas con suplementación (Toledano, 2008). Las diferencias entre los resultados aquí presentados y los estudios previos pueden ser debidas a: i) considerar además del ciervo a la cabra montés, ii) el alimento aportado a los animales no es de suficiente calidad proteica, y iii) el alimento no es suficiente para mitigar los efectos de la mayor densidad sobre la calidad de dieta. A la vista de la Figura 5 parece que las diferencias entre manejos se deben principalmente a la inclusión de la cabra, ya que los niveles de nitrógeno en las muestras de ciervo son bastante similares en ambos sistemas de manejo y elevadas en relación a otras fincas de similares características, por lo que parece que el efecto negativo de la mayor densidad de ciervos sobre la calidad de la dieta cuando los recursos son limitantes (ver Masahiko \& Keiji, 1999) queda mitigado con la suplementación.

En este mismo sentido, los resultados aquí presentados parecen indicar que la alimentación suplementaria no tiene efectos directos sobre la calidad de la dieta de las cabras monteses. De no ser así, y dada la ausencia de diferencias en densidad entre fincas, las cabras excretarían mayores niveles de 
nitrógeno en las fincas con alimentación suplementaria. Posiblemente la alimentación no esté disponible para las cabras debido a una elevada presión por parte de los ciervos, y el hecho de que los valores sean significativamente más elevados en las fincas sin manejo pudiera estar relacionado con cierta competencia entre especies dado el solapamiento de dieta existente. Por tanto, en los recorridos sin manejo, donde las densidades de ciervos son más reducidas, las cabras podrían ser más selectivas a la hora de seleccionar los recursos y por ello ingerir una dieta de mayor calidad proteica que los ciervos. Otras interpretaciones a estos resultados podrían buscarse en relación a la fisiología ruminal de ambas especies.

Respecto al ciclo estacional de los niveles de excreción de nitrógeno, los valores obtenidos en este estudio muestran un patrón lógico y coincidente con la productividad de estos ambientes. La primavera es el periodo donde mayores valores de nitrógeno se obtienen y el otoño y el invierno las que presentan los menores valores de productividad, y por tanto, de calidad de dieta. Los resultados obtenidos indican que para monitorizar los niveles de nitrógeno de una población en años sucesivos o de varias poblaciones, todas las muestras deben ser tomadas en un corto periodo de tiempo para evitar la variación estacional. La primavera al aportar el máximo valor del año se muestra el periodo más adecuado para ese propósito. Quizás muestrear en otoño-invierno pueda resultar también recomendable ya que indicarán la calidad de dieta mínima del año y será ésta la que pueda indicar la necesidad de establecer estrategias de gestión encaminadas a potenciarla.

\section{Implicaciones para la gestión}

Los estudios realizados sugieren que, para los indicadores biológicos empleados, las poblaciones de ciervo y cabra montés objeto de estudio muestran un adecuado estado poblacional. En este sentido sería esperable que la población de cabra montés continuara en ligero aumento en los próximos años a tenor de los indicadores obtenidos y de la percepción social sobre la tendencia de esta población.

El uso que las cabras monteses hacen del territorio podría estar determinado, en cierta medida, por la abundancia de ciervo y ésta, indirectamente, podría también estar determinando la calidad de la dieta de las monteses aunque por el momento no de forma alarmante. Por tanto, no sería esperable que el ciervo supusiera un riesgo relevante para la viabilidad de esta población de cabra montés, al menos si la gestión de las poblaciones de ciervos se mantiene como hasta el momento y las poblaciones no se disparan demográficamente. 
Finalmente, dado que las estrategias de gestión aplicadas en las fincas de estudio, centradas principalmente en potenciar las poblaciones de ciervo, parecen no tener efectos sobre la cabra montés, por lo que si fuese necesario aplicar alguna medida a la población de cabra, ésta tendría que hacerse de manera específica para esta especie, ya que de no ser así difícilmente estaría accesible para las cabras dada la elevada presión de los ciervos en estos territorios.

\section{Agradecimientos}

Este trabajo ha sido promovido y financiado por el Parque Nacional de Cabañeros (Organismo Autónomo de Parques Nacionales) en el marco del convenio de colaboración 8.06/5.46.3324. La realización de los trabajos de campo no hubiera sido posible sin la colaboración de Ángel Gómez y guías del Parque Nacional, Adolfo y Jesús de Parrillas y Francisco y Jorge de Muelas, muchas gracias por el apoyo logístico a pie de campo. Igualmente debemos agradecer la colaboración de "voluntarios" del IREC en los trabajos de campo, Luca, Ricardo, Alba, Virginia y Mónica... y también a Javi Urra de la UMA. Ya en el laboratorio el mérito es de Paqui Talavera en el preprocesado de las muestras para los análisis parasitológicos. P. Acevedo disfruta de un contrato dentro del programa Juan de la Cierva - Fondo Social Europeo.

\section{Referencias}

Acevedo, P. \& Cassinello, J., 2009a. Human-induced range expansion of wild ungulates causes niche overlap between previously allopatric species: red deer and Iberian ibex in mountainous regions of southern Spain. Annales Zoologici Fennici, 46: 39-50.

Acevedo, P. \& Cassinello, J., 2009b. Biology, ecology and status of Iberian ibex Capra pyrenaica: a critical review and research prospectus. Mammal Review, 39: 17-32.

Acevedo, P., Cassinello, J. \& Gortázar, C., 2007a. The Iberian ibex is under an expansion trend but displaced to suboptimal habitats by the presence of extensive goat livestock in central Spain. Biodiversity and Conservation, 16: 3361-3376.

Acevedo, P., Cassinello, J., Hortal, J. \& Gortázar, C., 2007b. Invasive exotic aoudad (Ammotragus lervia) as a major threat to native Iberian ibex (Capra pyrenaica): a habitat suitability model approach. Diversity and Distributions, 13: 587-597. 
EVALUACIÓN DEL ESTADO DE LA POBLACIÓN DE CABRA MONTÉS DE LOS MONTES DE TOLEDO:...

Acevedo, P., Ferreres, J., Jaroso, R., Durán, M., Escudero, M.A., Marco, J. \& Gortázar, C., 2010. Estimating roe deer abundance from pellet group counts in Spain: An assessment of methods suitable for Mediterranean woodlands. Ecological Indicators, 10: 1226-1230.

Acevedo, P. \& Real, R. en prensa. Biogeographycal differences between the two Capra pyrenaica subspecies, C. p. victoriae and C. p. hispanica, inhabiting in the Iberian Peninsula: conservation implications. Ecological Modelling.

Acevedo, P., Ruiz-Fons, F., Reyes-García, A.R. \& Gortázar, C., 2007c. Estudio comparativo de métodos para la estima de la abundancia de ungulados de montaña: la cabra montés. VIII Jornadas de la Sociedad Española para la Conservación y Estudio de los Mamíferos. Huelva.

Acevedo, P., Ruiz-Fons, F., Vicente, J., Reyes-García, A.R., Alzaga, V. \& Gortázar, C., 2008. Estimating red deer abundance in a wide range of management situations in Mediterranean habitats. Journal of Zoology, 276: 37-47.

Acevedo, P., Vicente, J., Alzaga, V. \& Gortázar, C., 2005. Relationship between bronchopulmonary nematode larvae and relative abundances of Spanish ibex (Capra pyrenaica hispanica) from Castilla-La Mancha, Spain. Journal of Helminthology, 79: 113-118.

Alasaad, S., Morrondo, P., Dacal-Rivas, V., Soriguer, R.C., Granados, J.E., Serrano, E., Zhu, X.Q., Rossi, L. \& Pérez, J.M., 2009. Brochopulmonary nematode infection of Capra pyrenaica in the Sierra Nevada massif, Spain. Veterinary Parasitology, 164: 340-343.

Anderson, R.C., 2000. Nematode parasites of vertebrates: their development and transmission. 672 pp. CABI Publishing Wallingford, Oxon.

Cordero del Campillo, M. \& Rojo, F.A., 1999. Parasitología veterinaria. Spain, McGraw Hill, Interamericana.

Demiaszkiewicz, A., 1987. Epizootiolgy of Elaphostrongylus cervi infection of red deer in the Bialowieza forest (in polish). Med Weter, 43: 208-211.

Edge, W.D. \& Marcum, C.L., 1989. Determining elk distribution with pelletgroup and telemetry techniques. Journal of Wildlife Management, 53: 621624.

Escós, J.M., Alados, C.L., Pulido, A., Romera, J., González-Sánchez, N. \& Martínez, F., 2008. Estimating population trends using population viability analyses for the conservation of Capra pyrenaica. Acta Theriologica, 53: 275-286.

Forrester, S.G. \& Lankester, M.W., 1997. Extracting Protostrongylid nematode from ungulate feces. Journal of Wildlife Diseases, 33: 511-516.

García-González, R. \& Herrero, J., 1999. El bucardo de los Pirineos: historia de una extinción. Galemys, 11: 17-26. 
Gordon, I.J., 1989. Vegetation community selection by ungulates on the Isle Rhum. II. Vegetation community selection. Journal of Applied Ecology, 26: 53-64.

Gortázar, C., Acevedo, P., Ruiz-Fons, F. \& Vicente, J., 2006. Disease risks and overabundance of game species. European Journal of Wildlife Research, 52: 81-87.

Gortázar, C., Herrero, J., Villafuerte, R. \& Marco, J., 2000. Historical examination of the distribution of large mammals in Aragón, Northeastern Spain. Mammalia 61: 411-422.

Hamel, S., Garel, M., Festa-Bianchet, M., Gaillard, J.M. \& Côté, S.D., 2009. Spring Normalized Difference Vegetation Index (NDVI) predicts annual variation in timing of peak faecal crude protein in mountain ungulates. Journal of Applied Ecology, 46: 582-589.

Hemami, M.R., Watkinson, A.R. \& Dolman, P.M., 2005. Population densities and habitat associations of introduced muntjac Muntiacus reevesi and native roe deer Capreolus capreolus in a lowland pine forest. Forest Ecology and Management 215: 224-238.

Martínez, T., Soriguer, R.C. \& Fandos, P., 1992. Trophics relationships between wild goat (Capra pyrenaica) and the red deer (Cervus elaphus) in the southeast Spain (S. Cazorla). En: Proceedings of the International Congress of the genus Capra in Europe: 225-228. Ronda, España.

Masahico, A. \& Keiji, O., 1999. Nitrogen content in feces and diet of sika deer on the Boso Peninsula, central Japan. Ecological Research, 14: 249-253.

Massey, B.N., Weckerly, F.W., Vaughn, C.E. \& Mccullough, D.R., 1994. Correlations between fecal nitrogen and diet composition in free-ranging black-tailed deer. The Southwestern Naturalist, 39: 165-170.

Mattson, W.J.J., 1980. Herbivory in relation to plant nitrogen content. Annual Review on Ecology and Systematics, 11: 119-161.

Melhorn, H., Düwel, D. \& Raether, W., 1992. Atlas de parasitologia veterinaria. Barcelona, Grass.

Moen, R., Pastor, J. \& Cohen, Y., 1997. Accuracy of GPS telemetry collar locations with differential correction. Journal of Wildlife Management, 61: 530539.

Pérez, J.M., Granados, J.E., Soriguer, R.C., Fandos, P., Márquez, F.J. \& Crampe, J.P., 2002. Distribution, status and conservation problems of the Spanish Ibex, Capra pyrenaica (Mammalia: Artiodactyla). Mammal Review, 32 : 26-39.

Putman, R.J., 1984. Facts from faeces. Mammal Review, 14: 79-97.

Shackleton, D.M., 1997. Wild Sheep and Goats and their Relatives: Status Survey and Conservation Action Plan for Caprinae. IUCN, Gland, Switzerland.

Smart, J.C.R., Ward, A.I. \& White, P.C.L., 2004. Monitoring woodland deer populations in the UK: an imprecise science. Mammal Review, 34: 99-114. 
Tayce, J., Acevedo, P., Vicente, J. \& Gortázar, C., 2008. Minimum sampling effort for reliable non-invasive estimations of excretion abundance of Elaphostrongylus cervi L1 in red deer (Cervus elaphus) populations. Journal of Helminthology, 82: 255-261.

Toledano, P., 2008. Relaciones entre la cantidad de nitrógeno fecal y características biométricas, de condición corporal, demográficas y de hábitat en poblaciones de ciervo (Cervus elaphus) del centro sur de España. Trabajo fin de Carrera, EUITA, Universidad de Castilla-La Mancha.

Vicente, J., Fernández-de-Mera, I.G. \& Gortázar, C., 2006. Epidemiology and risk factors analysis of elaphostrongylosis in red deer (Cervus elaphus) from Spain. Parasitology Research, 98: 77-85.

VIcente, J., Fierro, Y. \& Gortázar, C., 2005. Seasonal dynamics of the faecal excretion of Elaphostrongylus cervi (Nematoda, Metastrongyloidea) first stage larvae in Iberian red deer from South Spain. Parasitology Research, 95: 60-64.

Vicente, J., Höfle, U., Fernández-de-Mera, I.G. \& Gortázar, C., 2007. The importance of parasite life history and host density in predicting the impact of infections in red deer. Oecologia, 152: 655-664. 